\title{
The Chick Caudolateral Epiblast Acts as a Permissive Niche for Generating Neuromesodermal Progenitor Behaviours
}

\author{
Peter Baillie-Johnson ${ }^{\mathrm{a}, \mathrm{b}}$ Octavian Voiculescu ${ }^{\mathrm{c}}$ Penny Hayward ${ }^{\mathrm{b}}$ \\ Benjamin Steventon ${ }^{b}$ \\ a'Wellcome - Medical Research Council Cambridge Stem Cell Institute, University of Cambridge, Cambridge, UK;

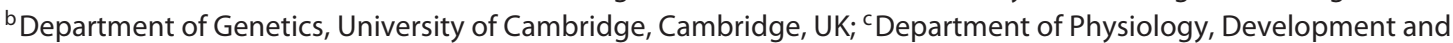 \\ Neuroscience, University of Cambridge, Cambridge, UK
}

\section{Keywords}

Neuromesodermal progenitors - Embryonic stem cells .

Presomitic mesoderm

\begin{abstract}
Neuromesodermal progenitors (NMps) are a population of bipotent progenitors that maintain competence to generate both spinal cord and paraxial mesoderm throughout the elongation of the posterior body axis. Recent studies have generated populations of NMp-like cells in culture, which have been shown to differentiate to both neural and mesodermal cell fates when transplanted into either mouse or chick embryos. Here, we aim to compare the potential of mouse embryonic stem (ES) cell-derived progenitor populations to generate NMp behaviour against both undifferentiated and differentiated populations. We define NMp behaviour as the ability of cells to: (i) contribute to a significant proportion of the anterior-posterior body axis, (ii) enter into both posterior neural and somitic compartments, and (iii) retain a proportion of the progenitor population within the posterior growth zone. We compare previously identified ES cell-derived NMp-like populations to undifferentiated
\end{abstract}

\begin{tabular}{ll}
\hline KARGER & $\begin{array}{l}\text { (c) } 2018 \text { The Author(s) } \\
\text { Published by S. Karger AG, Basel }\end{array}$ \\
$\begin{array}{l}\text { E-Mail karger@karger.com } \\
\text { www.karger.com/cto }\end{array}$ & $\begin{array}{l}\text { This article is licensed under the Creative Commons Attribution 4.0 } \\
\text { OpenAccenal License (CC BY) (http://www.karger.com/Services/ } \\
\text { Opense). Usage, derivative works and distribution are } \\
\text { permitted provided that proper credit is given to the author and the } \\
\text { original publisher. }\end{array}$
\end{tabular}

mouse ES cells and find that they all display similar potentials to generate NMp behaviour in vivo. To assess whether this competence is lost upon further differentiation, we generated anterior and posterior embryonic cell types through the generation of 3D gastruloids and show that NMp competence is lost within the anterior (Brachyury-negative) portion of the gastruloid. Together this suggests that in vitro-derived NMp-like cells maintain an ability to contribute to multiple germ layers that is also present within pluripotent ES cells, rather than acquiring a neuromesodermal competent state through differentiation.

(C) 2018 The Author(s)

Published by S. Karger AG, Basel

\section{Introduction}

Amniotes form the posterior tissues of their bodies progressively, from populations of progenitor cells situated within the posterior growth zone. Neuromesodermal progenitors (NMps) are one such population of posterior progenitors that continuously allocate cells to both the spinal cord and presomitic mesoderm (PSM) [Selleck and Stern, 1991; Brown and Storey, 2000; Tzouanacou et

Benjamin Steventon

Department of Genetics, University of Cambridge

Downing Street

Cambridge CB2 3EH (UK)

E-Mail bjs57@cam.ac.uk 
al., 2009; Henrique et al., 2015]. This ability to maintain multigerm layer competence past primary gastrulation stages presents a unique opportunity to understand the molecular mechanisms that maintain cellular competence during development [Steventon and Martinez Arias, 2017]. It could be that the ability to contribute to PSM and spinal cord compartments is a specific feature of NMps that is acquired upon differentiation into an NMplike state. Alternatively, one could consider the ability to generate both ectodermal and mesodermal derivatives as being a property of multipotent epiblast cells that is maintained until late stages within the NMp population. To distinguish between these possibilities, transplantation experiments are required that compare the ability of pluripotent and differentiated cells to contribute to multiple germ layers.

The functional properties of the embryonic NMp population have been demonstrated through transplantation experiments [Cambray and Wilson, 2002, 2007]. These experiments have demonstrated their capacity not only to contribute to neural and mesodermal tissues across many levels of the body axis, but also their ability to self-renew through serial heterochronic transplantation [Cambray and Wilson, 2002]. Embryonic NMps can therefore maintain their competence to form both neural and mesodermal tissues beyond the normal duration of somitogenesis, when introduced into a permissive niche such as that found in the earlier embryos. The common origin for these tissues was demonstrated conclusively through clonal labelling experiments that indicated the continued presence of a bipotent population throughout axial elongation in the mouse [Tzouanacou et al., 2009]. The patterns of clonal labelling predicted phases of expansion and depletion that have been confirmed subsequently by quantification of the number of Sox2 $2^{+}, \mathrm{T} /$ Brachyury ${ }^{+}$cells during axial elongation [Wymeersch et al., 2016].

The signals and transcription factors expressed in this embryonic domain are key features of protocols used to generate and maintain Sox 2 and T/Brachyury co-expressing cells in vitro [Gouti et al., 2014; Tsakiridis et al., 2014; Turner et al., 2014]. Treatment with the canonical Wnt signalling agonist CHIR99021 (Chiron) and exogenous FGFs are common features of these protocols. Tsakiridis et al. [2014] describe how, in cultures of mouse epiblast stem cells, the pulse of Wnt signalling acts to generate a mixture of committed mesendodermal progenitors and NMps from an initially primitive streakbiased subpopulation of the original culture [Tsakiridis et al., 2014]. The pulse of Chiron upregulates expression

Chick Caudolateral Epiblast as a

Permissive Niche for NMp Behaviours of Brachyury, as well as Hox genes from paralogous groups 5-9, which are active late in gastrulation. Similar results were obtained by Gouti et al. [2014, 2017], who described the formation of Brachyury and Sox 2 co-expressing cells in cultures of mouse embryonic stem (ES) cells that were grown in fibroblast growth factor 2 (FGF2) and treated with a pulse of Wnt signalling. We have shown that a pulse of Chiron, acting in concert with endogenous FGF signalling, can direct adherent cultures of mouse ES cells towards a neuromesodermal progenitorlike state [Turner et al., 2014, 2017; Beccari et al. 2018]. However, expression of Sox 2 and Brachyury is often accompanied by the expression of multiple other tissuespecific markers within these progenitor populations, suggesting that they resemble a mixed population of multipotent progenitors rather than a pure population of NMps [Edri et al., 2018].

In the absence of a definitive gene expression signature, transplantation to the mouse or chicken embryo has been used as a functional test of the capacity of in vitrodifferentiated cells to produce NMp-like contributions, similar to those described for the embryonic population [Cambray and Wilson, 2002, 2007; McGrew et al., 2008]. Transplantation into the mouse embryo is technically demanding due to its small size, its initially cup-shaped geometry and the internal nature of its development. Live imaging the incorporation of grafted cells adds an additional layer of complexity, so previous transplantations of in vitro-derived cells have been limited to endpoint analyses such as that reported by Tsakiridis et al. [2014]. The chicken embryo offers a more accessible host system for testing the in vivo potential of candidate NMp populations due to its large size and its external development. Gouti et al. [2014] showed that the grafted candidate NMps incorporate into both the neural tube and the somitic compartments of the chick. While these experiments demonstrate the competence of in vitro-derived Sox $2^{+}, \mathrm{T} /$ Brachyury $^{+}$populations to generate both neural and mesodermal derivatives in vivo, it is not clear whether this is an intrinsic property of NMp-like populations, or else a consequence of a multipotent population of cells responding to transplantation into a permissive niche. To address this, we set out to compare the ability of NMp-like cells to generate NMp behaviour to grafts of either uncommitted or more differentiated ES cells when transplanted into the chick caudolateral epiblast. We found that NMp-like cells show similar behaviours to pluripotent stem cells upon transplantation, while more differentiated populations are restricted in their ability to contribute to long portions of the embryonic body axis.

Cells Tissues Organs 2018;205:320-330 321 


\section{Materials and Methods}

\section{Chicken Embryo Culture}

Fertilised chicken eggs were stored in a humidified $10^{\circ} \mathrm{C}$ incubator for up to 1 week until required. The eggs were transferred to a humidified, rocking $37^{\circ} \mathrm{C}$ incubator for $24 \mathrm{~h}$ prior to the preparation of embryo cultures.

Embryo cultures were prepared according to a modified version of New Culture [New, 1955] using glass rings with rectangular cross-sections to support the explanted embryonic membranes within 35-mm-diameter bacterial Petri dishes (BD Falcon 351008), on a layer of thin albumen. Pannett-Compton saline was prepared to the formulation as described by Voiculescu et al. [2008]. Embryo cultures were incubated in a humidified $37^{\circ} \mathrm{C}$ incubator prior to use and were staged according to the Hamburger and Hamilton $(\mathrm{HH})$ series [Hamburger and Hamilton, 1992]. The embryos typically ranged from $\mathrm{HH} 4$ to $\mathrm{HH} 9$ after the 24-h incubation described above. Embryos were freshly fixed in $4 \%$ paraformaldehyde (v/v, in PBS) at the end of the incubation time.

\section{Fate Mapping}

Small quantities of CellTracker ${ }^{\mathrm{TM}}$ Red (C34552, Thermo Fisher Scientific; prepared in $20 \%$ sucrose [v/v]) were micropipetted onto the ectoderm around the node with a mechanically drawn capillary needle and a mouth pipette (Sigma-Aldrich, A5177), within a small droplet of Pannett-Compton saline. Two sites were labelled per embryo, on either side of the midline; the droplet of saline was aspirated immediately after the second label had been placed to remove excess dye.

\section{Mouse ES Cell Culture}

E14Tg2a [Hooper et al., 1987], T/Bra::GFP [Fehling et al., 2003] or Nodal ${ }^{\mathrm{HBE}-\mathrm{YFP}}$ [Papanayotou et al., 2014] ES cells were routinely cultured on $0.1 \%$ gelatine (Sigma-Aldrich G1890) precoated plastic and were maintained in ES+LIF medium $(500 \mathrm{~mL}$ Glasgow's Minimal Essential Medium [Gibco 11710-035], $5 \mathrm{~mL}$ sodium pyruvate [Invitrogen 11360-039], $5 \mathrm{~mL}$ non-essential amino acids [Gibco/Invitrogen 11140-035], 5 mL GlutaMAX [Gibco 35050038], $1 \mathrm{~mL} \beta$-mercaptoethanol [Gibco/Invitrogen 31350-010], 50 $\mathrm{mL}$ foetal bovine serum [FBS; Biosera FB-1090/500], $550 \mu \mathrm{L}$ leukaemia inhibitory factor [Recombinant LIF, produced in-house by

Fig. 1. The experimental schematic is shown (above). Embryonic cells caudal and lateral to the node show NMp-like behaviour and are sampled in chicken node explants. The location of the embryonic NMp population lateral to the node was determined through DiI tissue labelling (a-d) and confirmed by heterotopic transplantation of explanted node tissue (e-h). The starting position of each label was measured as the absolute axial and lateral position from the caudal limit of the node (the $x$ and $y$ axes, respectively, in $\mathbf{b}, \mathbf{c}$, $\mathbf{f}$ and $\mathbf{g}$; node at $[0,0])$. The length of the rostrocaudal spread of labelled cells was measured on each side of the midline and their contribution to the different tissue compartments was scored by inspection. $\mathbf{a}$ and $\mathbf{e}$ show the longest labelled cell contributions, in each case. $\mathbf{b}$ and $\mathbf{f}$ show the starting position of each label, coloured according to the final axial length of the labelled cell contribution: $<500 \mu \mathrm{m}$, blue; $500-1,750 \mu \mathrm{m}$, orange, and $>1,750 \mu \mathrm{m}$, red. c and g show the starting position of each label, coloured according to the Wellcome - Medical Research Council Cambridge Stem Cell Institute]). Cell cultures were maintained in humidified incubators at $37^{\circ} \mathrm{C}$ with $5 \% \mathrm{CO}_{2}$. Two-thirds of the culture medium was replaced daily with fresh, warm ES+LIF and the cultures were passaged into new flasks every $2-3$ days as required by enzymatic dissociation with $0.05 \%$ Trypsin-EDTA (Gibco 25300-054). Samples of ES cells used for grafting were cultured in ES+LIF and were collected 2 days after plating.

\section{Candidate NMp Differentiation}

Culture media used for differentiation or for gastruloid culture were prepared in a base medium of N2B27 (NDiff $227^{\circledR}$; Takara Bio, Y40002), supplemented with CHIR99021 (Chiron; Tocris Biosciences, 4423) and/or FGF2 (R\&D Systems, 3139-FB) as appropriate. Adherent cultures of candidate NMps were produced using the protocols described by Gouti et al. [2014] and Turner et al. [2014], respectively, and were collected $72 \mathrm{~h}$ after plating. Gastruloid cultures were prepared as described previously [Turner et al., 2017], either with or without Chiron treatment from 48 to $72 \mathrm{~h}$ after plating. Gastruloid tissues were dissected for grafting $120-125 \mathrm{~h}$ after plating.

\section{Preparation of Tissues for Grafting}

Adherent cells were detached mechanically using a cell scraper in PBS (with calcium and magnesium) to lift intact colonies with minimal sample dissociation. Enzymatic treatments, such as exposure to Accutase ${ }^{\circledR}$, were found to completely preclude cellular NMp-like behaviour after grafting (data not shown). The suspension was transferred to an FBS precoated FACS tube and was centrifuged at $170 \mathrm{~g}$ for $5 \mathrm{~min}$. The supernatant was discarded and the colonies washed by gentle resuspension in PBS (with calcium and magnesium) before the centrifugation was repeated. The colonies were resuspended in PBS (without calcium and magnesium; Sigma-Aldrich D8537) for labelling with DiI (Thermo Fisher Scientific Vybrant ${ }^{\circledR}$ V22885, 1\% v/v) for 25 min in the dark, on ice. The labelled colonies were centrifuged at $170 \mathrm{~g}$ for $5 \mathrm{~min}$ and the pellet was resuspended in $37^{\circ} \mathrm{C} \mathrm{PBS}$ (with calcium and magnesium) for grafting.

Gastruloid tissues were collected with a micropipette and were dissected into small pieces using a hair loop tool and an eyebrow knife in warm N2B27. Dissected tissues were transferred to an FBS whether the labelled cells contributed to neural tissues (purple), mesodermal tissues (green) or both (orange). Contributions to the anterior tissues of the head are indicated in grey in c. A rectangular ROI (dashed line in $\mathbf{b}, \mathbf{c}, \mathbf{f}$ and $\mathbf{g}$ ) is defined as the largest region that encompasses all of the long, mixed neural and mesodermal contributions. The Venn diagrams (d, h) summarise the overall behaviour of the labels or grafts. Each label/graft was scored into the three sets, corresponding to long contributions, mixed neural and mesodermal contributions, and cases where labelled cells resided in the region around the node. The percentage of grafts satisfying all three conditions is shown in the bottom right. The number of single-germ layer restricted, short or medium labels/grafts that did not occupy the region around the node is indicated below the plot. $n$ denotes the number of labels/grafts, and $N$ the number of biological replicates. 
Experimental schematic
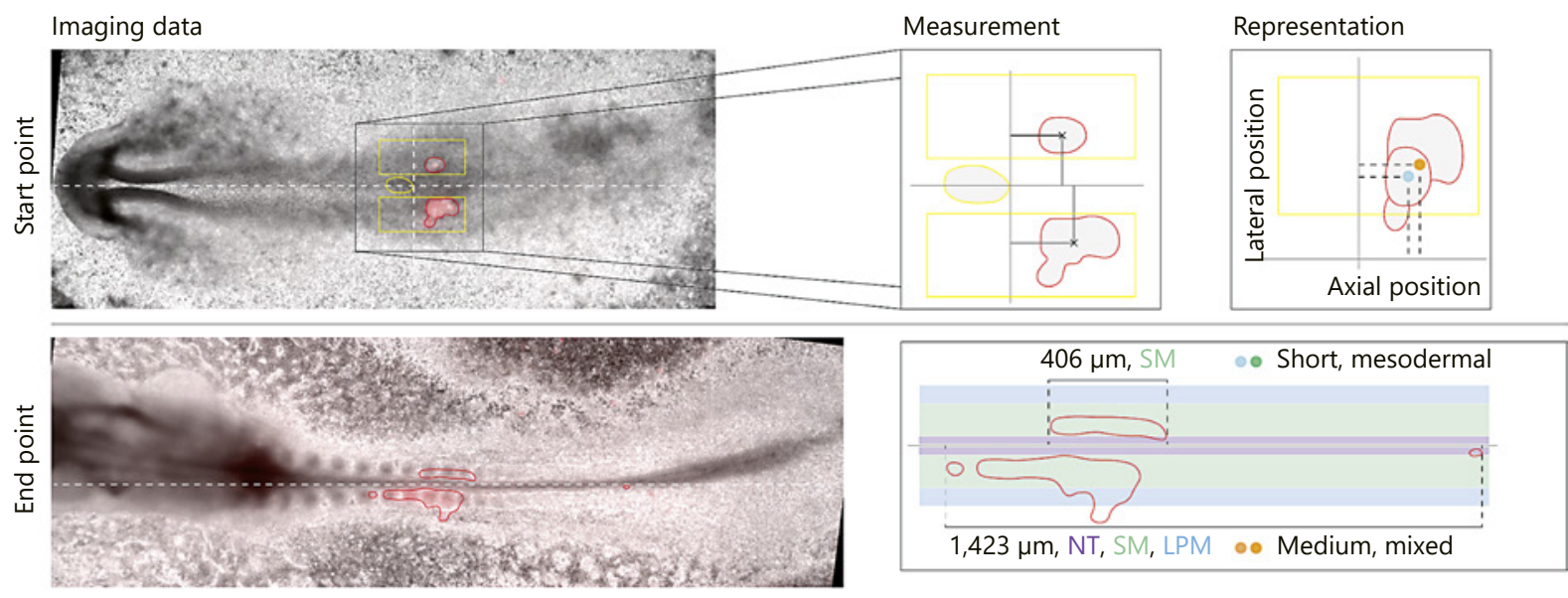

Dil fate mapping ( $n=66, N=2)$

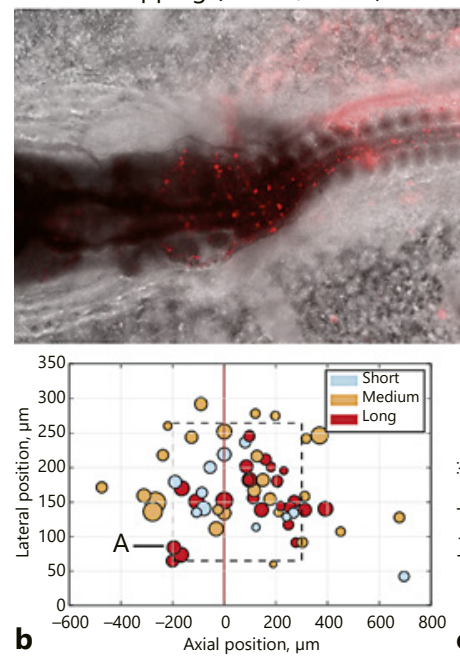

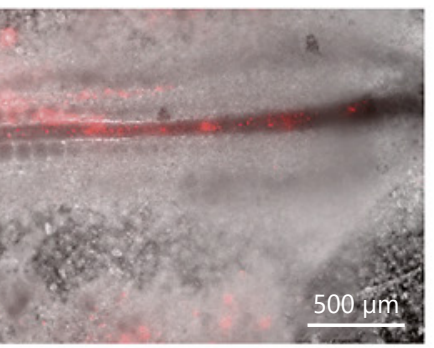

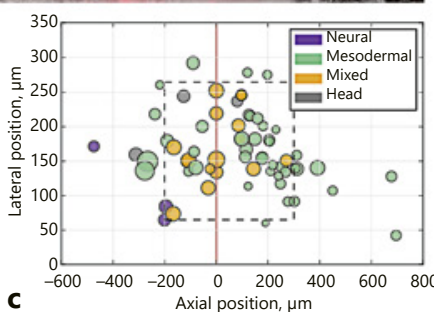

Fate mapping

$>1,750 \mu \mathrm{m}$

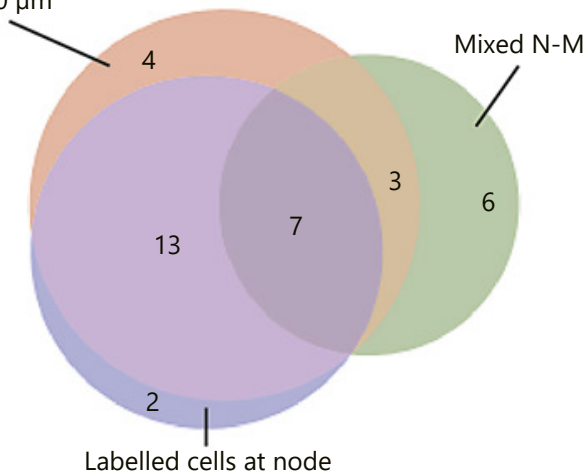

31

d $n=66$

Chick node explants $(n=24, N=2)$
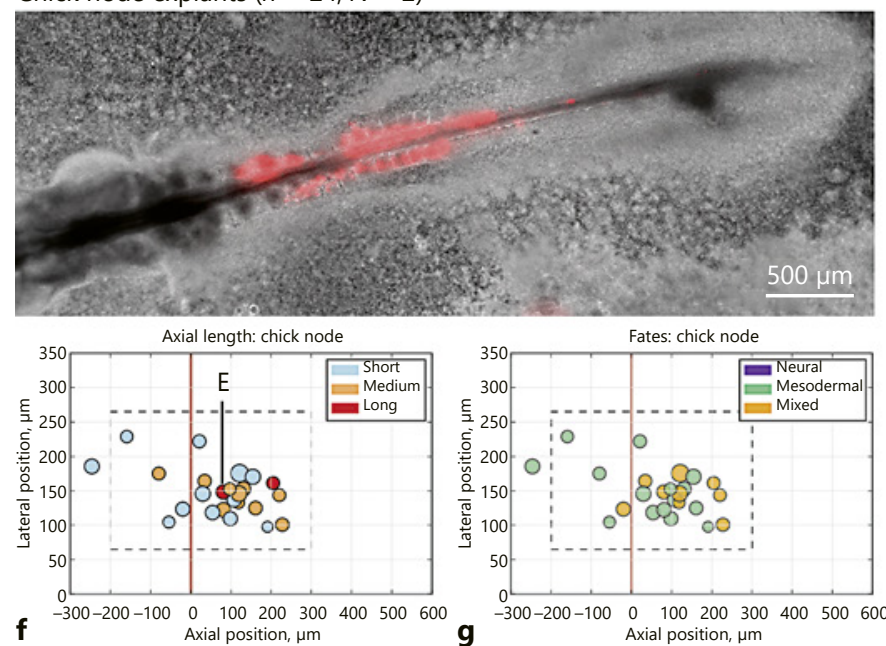

Chick node

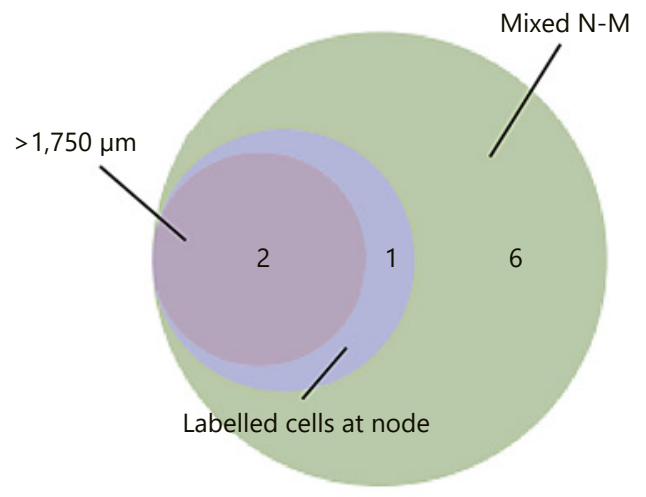

15

$8 \%$

Chick Caudolateral Epiblast as a

Permissive Niche for NMp Behaviours
Cells Tissues Organs 2018;205:320-330 DOI: $10.1159 / 000494769$ 
precoated FACS tube and were labelled as above. Explants of embryonic tissue from a square region around the node were dissected with a tungsten needle or an eyebrow knife and were labelled as above.

\section{Grafting Procedure}

Any embryos that were developing abnormally or had flooded with albumen were discarded prior to grafting. A drop of PannettCompton saline was pipetted onto the surface of the embryo and two labelled fragments were transferred into the droplet with a mouth pipette. An eyebrow knife tool or an electrolytically sharpened tungsten needle [Brady, 1965] was used to make a small opening in the ectoderm caudal and lateral to the node on each side of the midline. The labelled fragment was positioned in this opening using the tool and the droplet of saline was aspirated to remove any ungrafted labelled cells. The lid of each culture dish was sealed with albumen and the culture was returned to the incubator to heal briefly prior to imaging. Every culture was imaged (see below) within an hour of grafting and approximately $18 \mathrm{~h}$ of grafting; a subset of six embryos was also imaged overnight at 20-min intervals with time-lapse microscopy in each experiment.

\section{Microscopy}

Widefield, single time points and time-lapse images were acquired with a Zeiss AxioObserver Z1 (Carl Zeiss, UK) using a $5 \times$ objective in a humidified $37^{\circ} \mathrm{C}$ incubator, with the embryo cultures positioned on the inverted lid of a six-well plate. An LED white light illumination system (Laser 2000, Kettering, UK) and a Filter Set 45 filter cube (Carl Zeiss, UK) was used to visualise red fluorescence. Emitted light was recorded using a back-illuminated iXon888 Ultra EMCCD (Andor, UK) and the open source MicroManager software (Vale Lab, UCSF, USA).

\section{Quantification}

The open-source FIJI ImageJ platform [Schindelin et al., 2012] and the pairwise stitching plugin [Preibisch et al., 2009] were used for image analysis. Any embryos that were developing abnormally or where the grafted cells had become lost were excluded from further analysis. Each set of images was scored for size and starting position of each graft in relation to the medio-caudal limit of the node, the tissues to which the labelled cells contributed and the final distance between the most rostral and most caudal cells on one side of the midline at the endpoint (around $18 \mathrm{~h}$ after grafting).

Fig. 2. ES cells (ESCs) and ESC-derived NMps are competent to produce NMP-like contributions on transplantation. Labelled ESCs (i-I) and ESC-derived NMps (a-d and $\mathbf{e - h}$ ) were transplanted into the region caudal and lateral to the node. The starting position of each graft was measured as the absolute axial and lateral position from the caudal limit of the node (the $x$ and $y$ axes, respectively, in $\mathbf{b}, \mathbf{c}, \mathbf{f}, \mathbf{g}, \mathbf{j}$ and $\mathbf{k}$; node at $[0,0])$. The length of the rostrocaudal spread of labelled cells was measured on each side of the midline and their contribution to the different tissue compartments was scored by inspection. $\mathbf{a}, \mathbf{e}$ and $\mathbf{i}$ show the longest labelled cell contributions for each cell culture. $\mathbf{b}, \mathbf{f}$ and $\mathbf{j}$ show the starting position of each graft, coloured according to the final length of the labelled cell contribution: $<500 \mu \mathrm{m}$, blue; $500-1,750 \mu \mathrm{m}$, orange,
Any grafts that were initially placed wholly outside the region of interest (ROI) were excluded from further analysis (online suppl. Fig. 11; for all online suppl. material, see www.karger.com/ doi/10.1159/000494769).

Measurements were compiled in Microsoft Excel and were plotted in Python 2.0 with the open source Project Jupyter iPython Notebook and the Matplotlib library. Box plots were prepared in R using the BoxPlotR webtool (Tyers and Rappsilber Labs, Université de Montréal, Canada, and University of Edinburgh, UK; respectively), following the conventions described in the accompanying primer [Krzywinski and Altman, 2014]. Venn diagrams were produced in Python 2.0 with the Matplotlib-venn 0.11.5 package.

\section{Results}

\section{The Region Lateral to the Node Contributes}

Extensively to Both Neural and Mesodermal Tissue

To identify a region of the embryo in which to graft candidate populations of neuromesodermal progenitors, a fate map was constructed by labelling small areas of the ectoderm with DiI (see Materials and Methods). The position of the labelled cells was recorded in relation to the caudal limit of the node after labelling and the size of the label was measured in the rostrocaudal (hereafter "axial") direction. After $15 \mathrm{~h}$ of incubation, the distance between the most rostral and most caudal labelled cells was measured and is shown in Figure $1 \mathrm{~b}$ (and online suppl. Figure 2A). The contribution of the labelled cells to the different tissue compartments was determined (online suppl. Fig. 2B) and is summarised in Figure 1c. On mapping the labels in this way, it is clear that labelled cells positioned in a small ROI lateral and slightly caudal to the node (shown as a dashed box in Fig. 1-3 and online suppl. Fig. 2-9) can contribute extensively to both neural and mesodermal tissues (a "mixed" contribution), as is consistent with previously published fate maps of this region

and $>1,750 \mu \mathrm{m}$, red. $\mathbf{c}, \mathbf{g}$ and $\mathbf{k}$ show the starting position of each graft, coloured according to whether the labelled cells contributed to neural tissues (purple), mesodermal tissues (green) or both (orange). The Venn diagrams $(\mathbf{d}, \mathbf{h}, \mathbf{I})$ summarise the overall behaviour of the grafts. Each graft was scored into the three sets, corresponding to long contributions, mixed neural and mesodermal contributions, and cases where labelled cells resided in the region around the node. The percentage of grafts satisfying all three conditions is shown in the bottom right. The number of single-germ layer restricted, short or medium grafts that did not occupy the region around the node is indicated below the plot. $n$ denotes the number of grafts, and $N$ the number of biological replicates.

(For figure see next page.) 

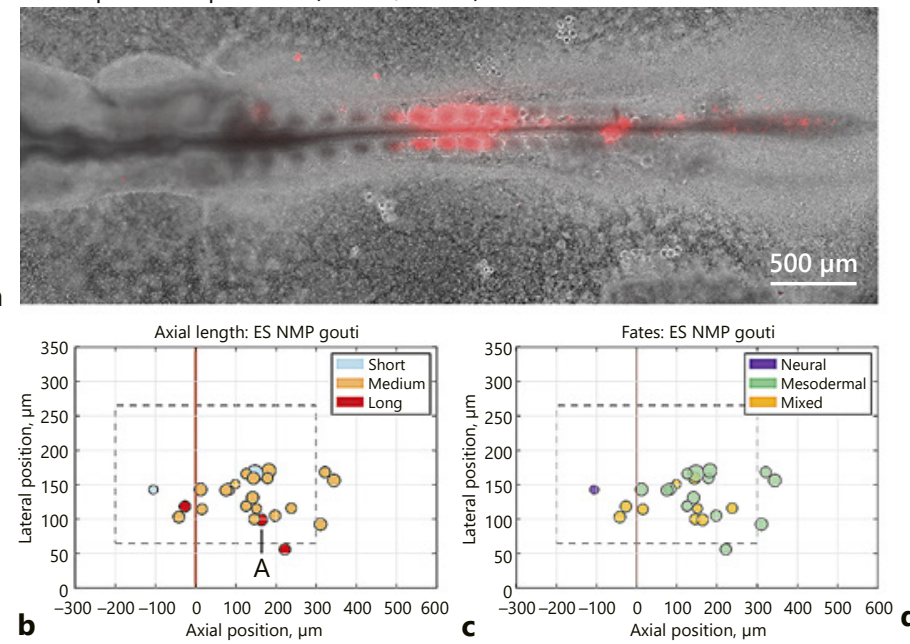

ES NMp - Turner protocol $(n=24, N=3)$
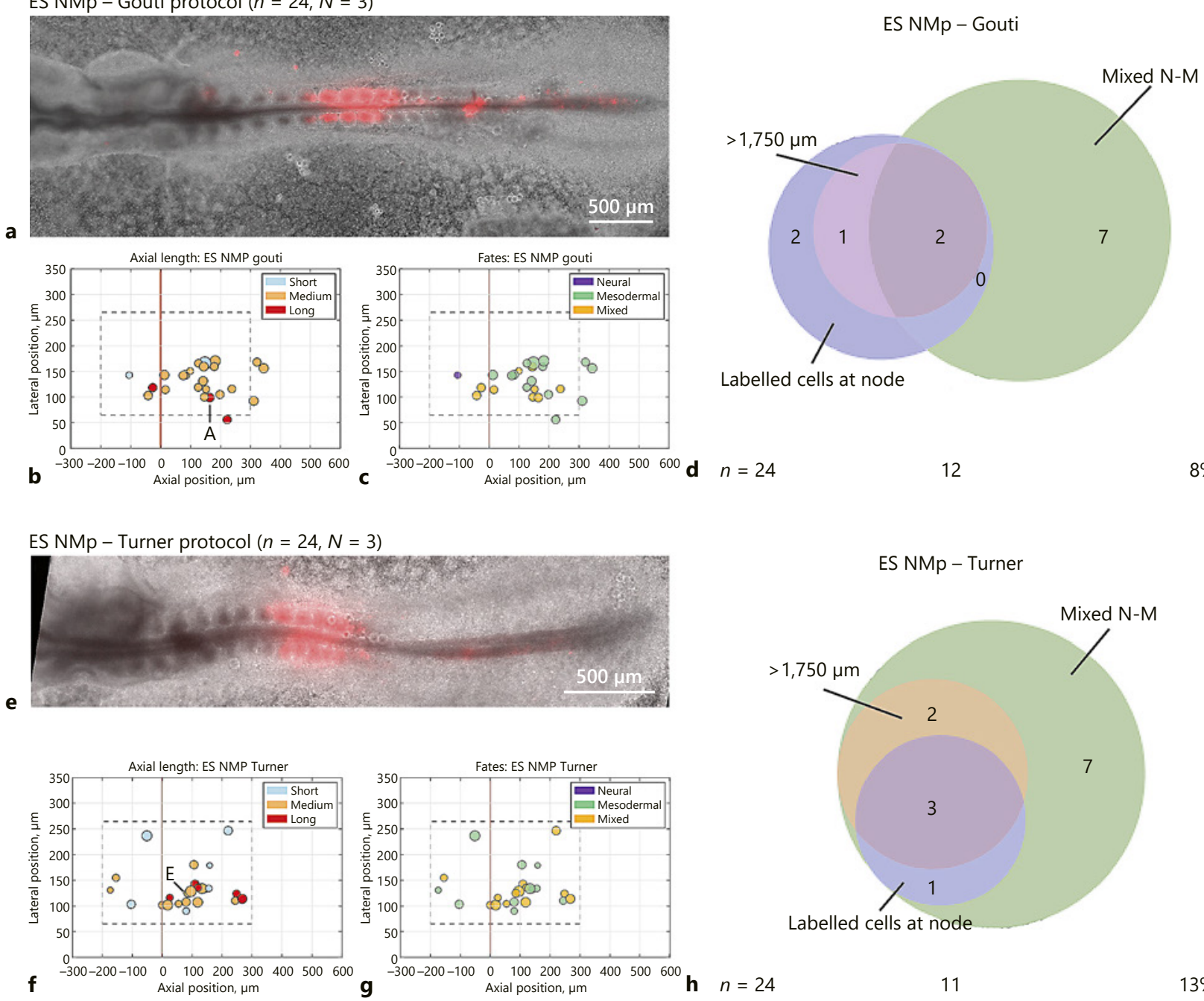

Labelled cells at node

ES NMp - Turner

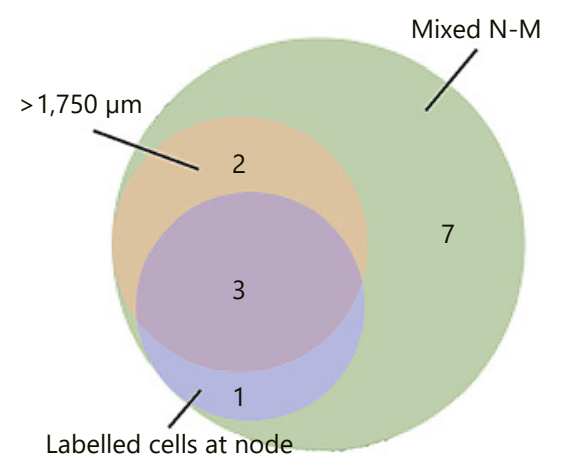

11

$\operatorname{ESCS}(n=11, N=1)$

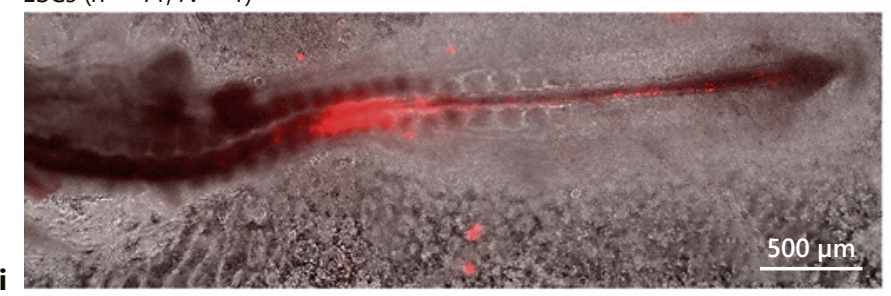

ESCs
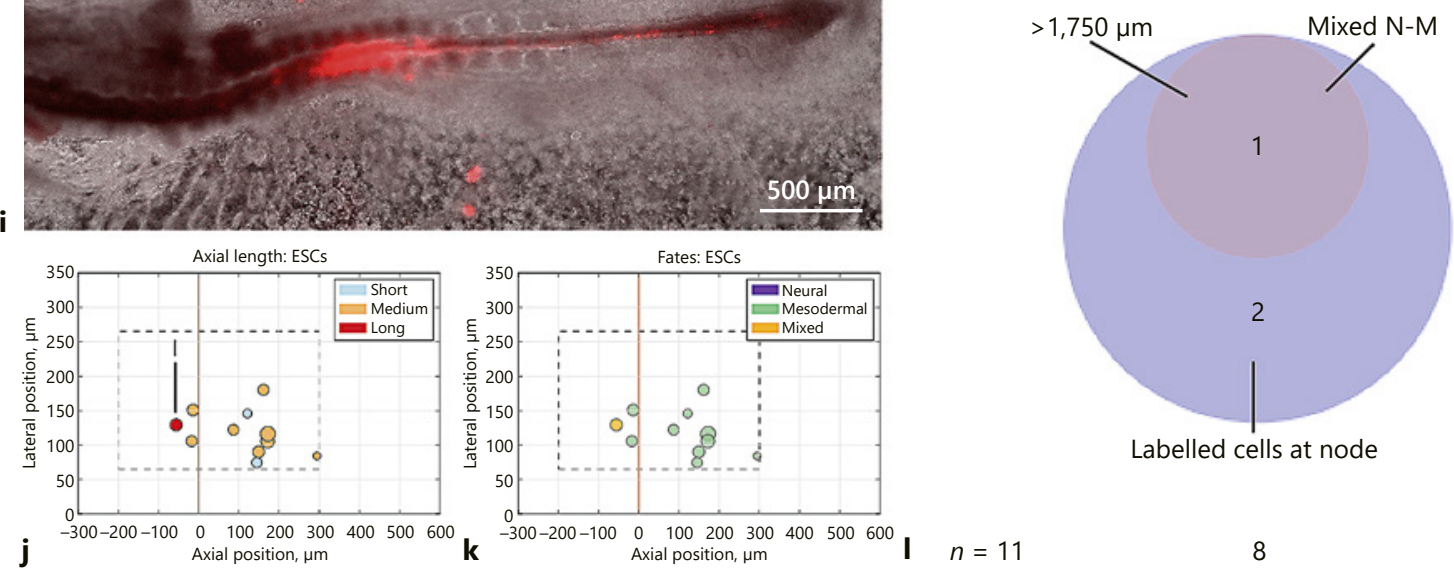

Labelled cells at node

8 
[Selleck and Stern, 1991; Catala et al., 1996; Brown and Storey, 2000]. In order to discriminate between axial contributions of different lengths, a series of thresholds were used: a lower one at $500 \mu \mathrm{m}$, which is less than the length of three somites, and an upper threshold of $1,750 \mu \mathrm{m}$ (online suppl. Fig. 1). Where the distance between the most rostral and most caudal labelled cells was less than the lower threshold, the contribution was defined as "short"; between the thresholds was defined as "medium" and above the upper threshold was defined as "long."

By further considering which tissues were occupied by the labelled cells, this ROI was found to include all of the long contributions to both neural and mesodermal tissues. It was noted that some of the long, mixed contributions also included cells resident in the region around the node at the end of the incubation period ( $11 \%$ of $66 \mathrm{grafts}$; Fig. 1d; red points in online suppl. Fig. 2). When cells were labelled rostrally to this ROI, they could contribute exclusively to neural tissues, while labels placed more caudally were exclusively mesodermal (Fig. 1c). The results of the fate mapping therefore define a region in which to graft populations of candidate NMps in order to test their capacity to contribute to neural and mesodermal tissues across many axial levels.

As a positive control for the generation of NMps in this assay, we labelled explants of the region around and including the chicken node and grafted them into the region defined by the fate mapping experiment. When grafted into the ROI, the explants were found to give mostly mesodermal and mixed contributions that were infrequently long (Fig. 1e-h; online suppl. Fig. 3). Timelapse imaging showed that the long contributions arose through labelled cells remaining in the region around the node as it regressed, with some cells leaving to occupy the PSM (online suppl. Movie 1). The results of grafting the node explants show that a candidate neuromesodermal

Fig. 3. Anterior explants from Chiron-treated gastruloids are not competent to produce NMp-like behaviour, unlike posterior or untreated explants. Labelled tissue explants from Chiron-treated (anterior, a-d, or posterior, $\mathbf{e - h}$ ) or untreated (i-I) gastruloids were grafted into the region caudal and lateral to the node. The starting position of each graft was measured as the absolute axial and lateral position from the caudal limit of the node (the $x$ and $y$ axes, respectively, in $\mathbf{b}, \mathbf{c}, \mathbf{f}, \mathbf{g}, \mathbf{j}$ and $\mathbf{k}$; node at $[0,0]$ ). The length of the rostrocaudal spread of labelled cells was measured on each side of the midline and their contribution to the different tissue compartments was scored by inspection. $\mathbf{a}$, e and $\mathbf{I}$ show the longest labelled cell contributions in each case. $\mathbf{b}, \mathbf{f}$ and $\mathbf{j}$ show the starting position of each graft, coloured according to the final length of the progenitor population would be expected to mimic this pattern of NMp-like behaviour in producing long mixed contributions at an equal or higher frequency $(\geq 8 \%$, with the number of grafts within the NMp-fated domain being $\left.\mathrm{n}_{\mathrm{ROI}}=23\right)$.

\section{ES Cells and ES Cell-Derived Candidate NMps Are Competent to Show NMp-Like Behaviour on Transplantation}

To further test the suitability of this assay for testing murine ES cell-derived NMps, candidate cells were produced in accordance with published protocols and were transplanted to the region caudal and lateral to the node. Any grafts that were initially placed wholly outside this region were excluded from further analysis (online suppl. Fig. 11). Cells cultured according to the protocol used by Gouti et al. [2014] have previously been described to contribute to both neural and mesodermal tissues. In this assay, these cells were found to produce mostly mixed and mesodermal contributions, some of which were long (Fig. 2a-d; online suppl. Fig. 4). The frequency of long, mixed contributions within the ROI was found to be $10.00 \%\left(n_{\text {ROI }}=20\right)$. Grafts were also observed to contribute labelled cells to the PSM and to the region around the node.

Candidate NMps generated with the protocol published by Turner et al. [2014, 2017] have not previously been tested in vivo. In this assay, they were found to give mostly mixed and mesodermal contributions, some of which were long (Fig. 2e-h; online suppl. Fig. 5). The long, mixed contributions occurred at a frequency of $20.83 \%\left(\mathrm{n}_{\mathrm{ROI}}=24\right)$ and included labelled cells in the region of the node and in the PSM (online suppl. Fig. 5). In a manner similar to the grafted node explants, the labelled cells were observed in time-lapse experiments to transiently reside in the region around the node during its

labelled cell contribution: $<500 \mu \mathrm{m}$, blue; $500-1,750 \mu \mathrm{m}$, orange and $>1,750 \mu \mathrm{m}$, red. $\mathbf{c}, \mathbf{g}$ and $\mathbf{k}$ show the starting position of each graft, coloured according to whether the labelled cells contributed to neural tissues (purple), mesodermal tissues (green) or both (orange). The Venn diagrams in $\mathbf{d}, \mathbf{h}$ and $\mathbf{I}$ summarise the overall behaviour of the grafts. Each graft was scored into the three sets, corresponding to long contributions, mixed neural and mesodermal contributions, and cases where labelled cells resided in the region around the node. The percentage of grafts satisfying all three conditions is shown in the bottom right. The number of single-germ layer restricted, short or medium grafts that did not occupy the region around the node is indicated below the plot. $n$ denotes the number of grafts, and $N$ the number of biological replicates.

(For figure see next page.) 

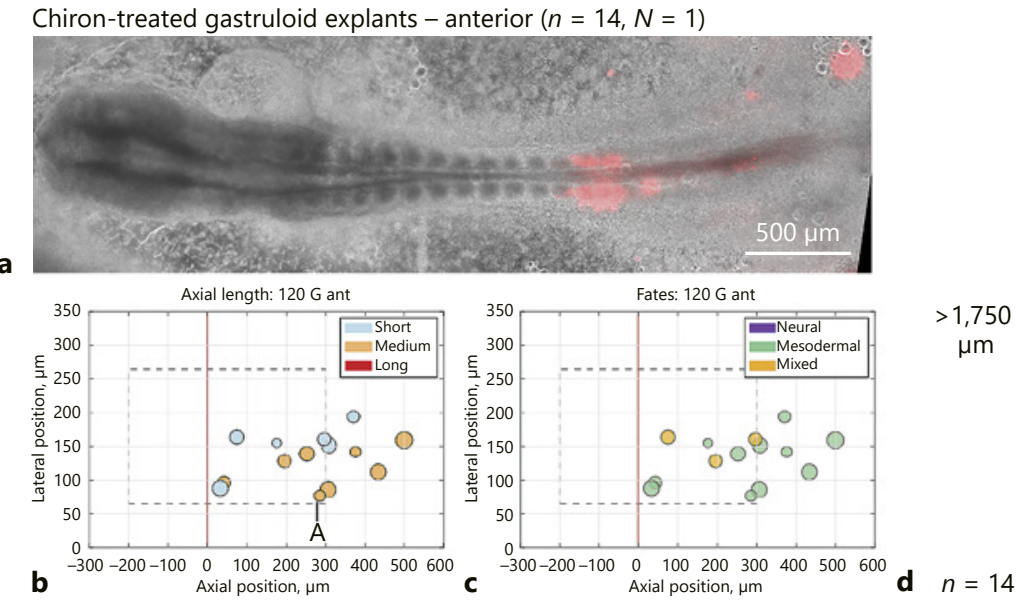

Anterior gasts Axial position, $\mu \mathrm{m}$

Chiron-treated gastruloid explants - posterior $(n=19, N=2)$
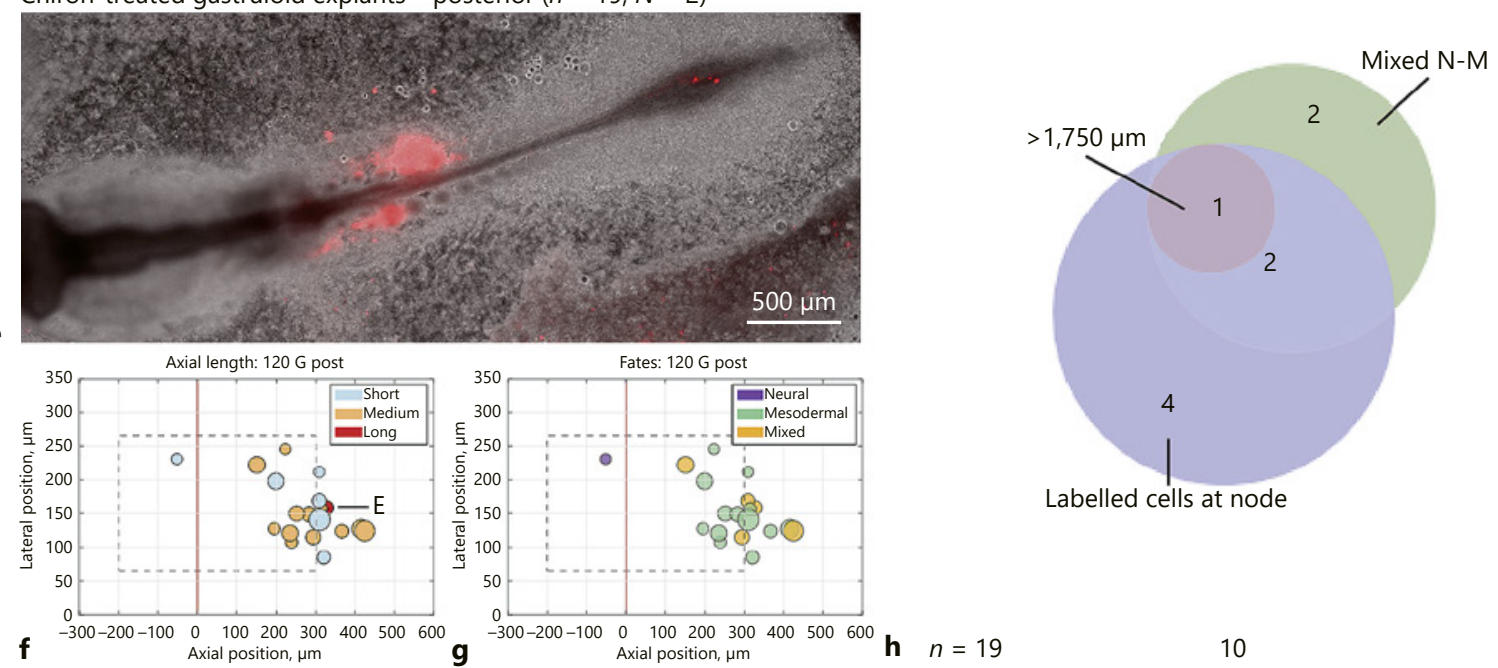

Labelled cells at node

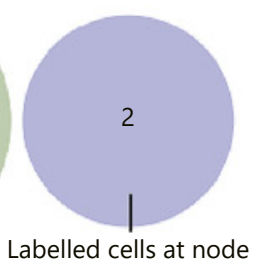

Mixed N-M

N2B27-cultured gastruloid explants $(n=25, N=1)$
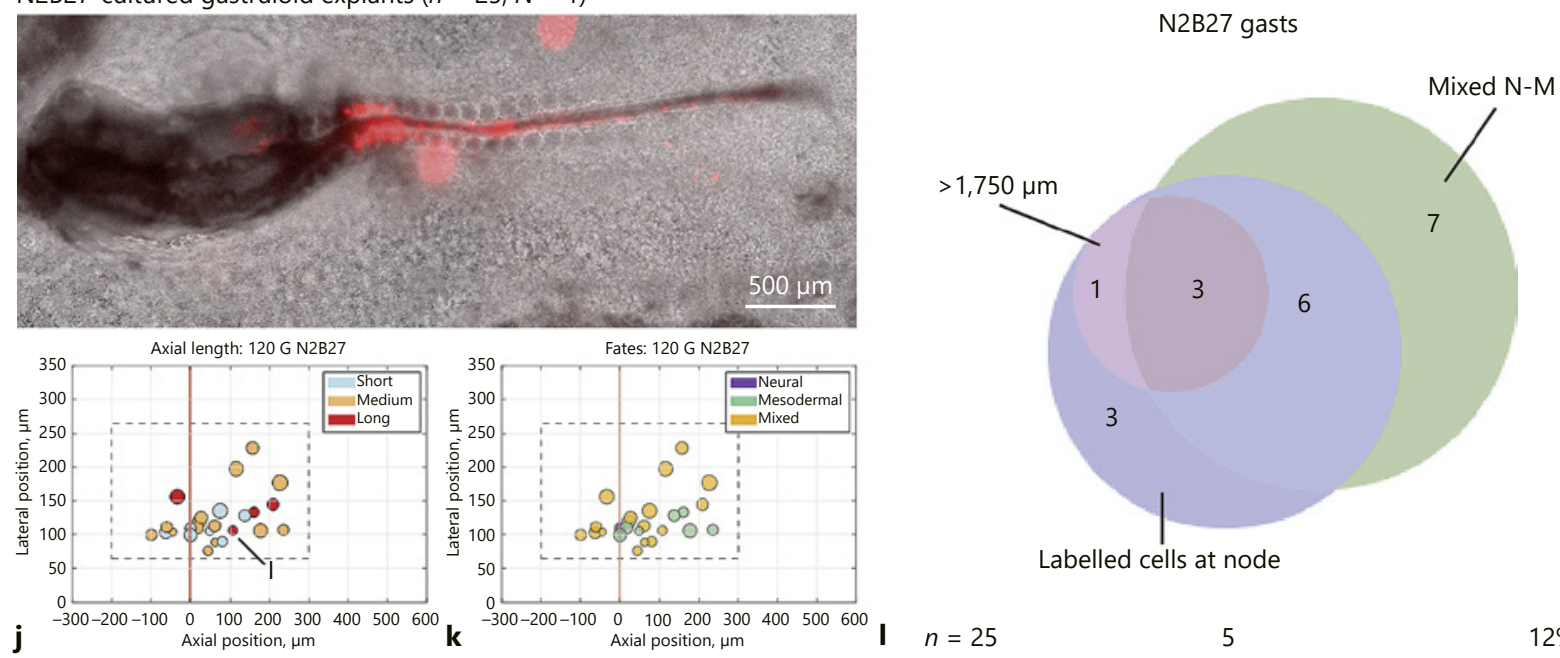

Fates: 120 G N2B27

Labelled cells at node

5 
regression (online suppl. Movie 2). Collectively, these results demonstrate that murine ES cells cultured according to both protocols are competent to show NMp-like behaviour on transplantation to the region caudal and lateral to the node. These observations validate the use of this assay as these populations have been described previously as NMp competent.

On grafting self-renewing cultures of ES cells, however, a similar pattern of long, neural and mesodermal contribution was observed at a frequency of $9.09 \%$ $\left(\mathrm{n}_{\mathrm{ROI}}=11\right.$; Fig. $\left.2 \mathrm{i}-\mathrm{l}\right)$ after transplantation to the same region. These grafts appeared to integrate efficiently into the embryonic tissues, with every graft appearing to spread in some way during the incubation. One graft was observed to produce a long, mixed contribution that included labelled cells in the region around the node (Fig. 2l), while the remaining ten grafts contributed to limited extents of the mesodermal tissues (online suppl. Fig. 6). Therefore, at the level of cell integration and anterior-posterior spreading along the embryonic axis, mouse ES cells behave in a similar manner to heterotopic and heterochronic grafts from chick embryos. This would suggest that the behaviours observed for NMp differentiation from ES cells reflect the maintenance of an earlier characteristic of pluripotent cells, rather than acquiring an NMp competent state through differentiation.

\section{Anterior Explants from Chiron-Treated Gastruloids}

Are Not Competent to Produce NMp-Like Behaviour,

Unlike Posterior or Untreated Explants

In order to test whether this assay can identify cells that are not competent to show NMp-like behaviour, more differentiated cultures were transplanted to the region caudal and lateral to the node. Three-dimensional gastruloid cultures were selected for this purpose, due to the fact that they develop a clear anterior-posterior polarity [Turner et al., 2017] with a candidate NMp population thought to reside in the more posterior tissues [Turner et al., 2014]. The anterior tissues are thought to be mostly mesodermal and locally express genes such as GATA6, thought to indicate a population of precardiac mesoderm [Turner et al., 2017]. The anterior-posterior polarity of Chiron-treated gastruloids can be scored reliably through phase-contrast microscopy alone [Baillie-Johnson, 2017], allowing them to be dissected into corresponding tissue explants prior to transplantation.

On grafting the anterior tissue explants to the ROI, $13 / 16$ of the grafts were observed to give short- to medium-length mesodermal contributions (Fig. 3b, c), including labelled cells in the PSM and, in 1 case, the region around the node (online suppl. Fig. 7). The remaining three grafts produced mixed contributions, though none of these was long. These tissues clearly lack the competence to produce NMp-like behaviour when grafted into this region of the embryo and time-lapse movies reveal how cells retain next to the node and only generate short axial contributions (online suppl. Fig. 3). In contrast, the posterior gastruloid tissues additionally produced a long, mixed neural and mesodermal contribution, indicating that a subpopulation of these cells retains the competence to show NMp-like behaviour in this assay. Many of the grafts were observed to contribute to the PSM and to the region around the node (online suppl. Fig. 8) at a higher frequency than for the anterior explants (40 vs. $12.5 \% ; n=19$ and 14 , respectively). The frequency of NMp-like contributions appears to be around half of that observed for the chick node explants, ES cells and ES cellderived NMps, suggesting that the more differentiated gastruloid tissues have more limited competence in this assay.

In order to test this idea further, tissue explants from gastruloids that had not been exposed to Chiron were tested in this assay. Gastruloids cultured in N2B27 alone develop entirely under the influence of endogenous signalling, so likely represent a less differentiated population compared to those treated with Chiron, which promotes symmetry-breaking and axial elongation. On grafting these tissues, the labelled cells produced mostly mixed contributions, which were long at a frequency of $12 \%$ $\left(\mathrm{n}_{\text {ROI }}=25\right.$; Fig. 3i-l $)$. Time-lapse imaging showed that labelled cells often left the main part of the graft to reside in the region around the node as it regressed (52\% of 25 cases; online suppl. Movie 4), though labelled cells were never found in the PSM (online suppl. Fig. 9). Collectively, the frequency of long mixed contributions indicates that these explants behave like the 2D culture-derived candidate neuromesodermal progenitor populations (Fig. 2). Given the higher frequency of mixed contributions, this culture produces cells with a greater propensity for neural differentiation than following the Chiron treatment. It therefore seems that the N2B27-cultured gastruloid explants are more frequently competent to show NMp-like behaviour on transplantation to the region caudal and lateral to the node.

In order to test whether the lengths of the axial contributions from the candidate neuromesodermal progenitor populations differ from those observed for pluripotent cells, the length measurements were plotted as box and whisker diagrams (online suppl. Fig. 10). When presented in this way, it is clear that all of the 
populations tested, with the exception of the anterior Chiron-treated gastruloids, are capable of producing long, mixed contributions after transplantation (online suppl. Fig. 10C).

\section{Discussion}

Taken together, these results show that the chick caudolateral epiblast is a permissive environment for competent cells to contribute to neural and mesodermal compartments of the elongating body axis. Under such conditions, derivatives of the grafted populations will be exposed to the appropriate signals for the specification to either cell fate. Therefore, this acts as an appropriate assay to assess the competence of cells to generate neural and mesodermal derivatives in vivo. This is important, as in many ways NMps could be considered as a population of cells that retain an epiblast-like state until later developmental stages, whose competence to generate different cell types is gradually lost as development proceeds. Transplantation assays such as the one presented here are therefore an appropriate method to test the state of NMps taken from the embryo, or NMp-like cell populations generated in culture.

Paraxial mesoderm progenitors have been shown to change their timing of entry into the primitive streak in a Hox-dependent manner [Iimura and Pourquié, 2006; Denans et al., 2015]. Whether this timing is reflected in alterations of the autonomous state of NMps as they undergo maturation into more posteriorly fated cells is unknown. The results presented here highlight transplantations into the caudolateral epiblast as an appropriate experimental assay to explore this as later differentiated NMp populations might be expected to delay their entry into the body axis as compared to earlier, more epiblastlike cell states. Indeed, this is something we have observed when comparing NM-like cells that have been maintained in culture for longer time periods [Edri et al., 2018]. The exact nature of this mechanism has yet to be explored.

As a culture of pluripotent cells, it is not surprising that the ES cell grafts were competent to make continued contributions to both neural and mesodermal tissues when grafted into a region that is permissive for the embryonic progenitor population. This may arise through responses to the signalling environment at the graft site and morphogenetic movements such as tissue flows that also distribute cells along the rostrocaudal axis, giving the impression of a contribution to axial elongation. Thus, although this assay allows this behaviour to be demonstrated for a given population, it does not define a population of NMps as a specific cell type, as many populations may be competent to behave in the same way when transplanted to this region. Nevertheless, the results from this assay show that more differentiated cells (such as the anterior gastruloid explants) cannot be made to behave like NMp-competent populations on grafting to the caudal lateral epiblast. Thus, we propose that the power of this experimental assay lies not in a positive test for a candidate NMp population, but rather for asking when cells are no longer competent to contribute to multiple germ layers during development.

\section{Acknowledgements}

Thanks are due to Dr. David Turner for his assistance in developing the image analysis pipeline and to Prof. Alfonso Martinez Arias for his role in devising the study.

\section{Disclosure Statement}

The authors declare no competing financial interests.

\section{Funding Sources}

B.S. was funded by a Sir Henry Dale Fellowship jointly funded by Wellcome and the Royal Society (109408/Z/15/Z). P.B.-J. was supported by an Engineering and Physical Sciences Research Council (EPSRC) Studentship (1359454). OV was funded by Wellcome Fellowship (RCDF 088380/09/Z).

\section{Author Contributions}

P.B.-J. and B.S. devised the study and wrote the manuscript. P.B.-J., O.V. and B.S. conducted the experiments. P.B.-J. acquired, quantified and analysed the imaging data. 


\section{References}

Baillie-Johnson, P. (2017) The generation of a candidate axial precursor in three dimensional aggregates of mouse embryonic stem cells; thesis, University of Cambridge. DOI: 10.17863/CAM.13742.

Beccari, L., N. Moris, M. Girgin, D.A. Turner, P. Baillie-Johnson, A.C. Cossy, M.P. Lutolf, D. Duboule, A. Martinez Arias (2018) Multi-axial self-organization properties of mouse embryonic stem cells into gastruloids. Nature 562: 272-276.

Brady, J. (1965) A simple technique for making very fine, durable dissecting needles by sharpening tungsten wire electrolytically. Bull World Health Org 32: 143-144.

Brown, J.M., K.G. Storey (2000) A region of the vertebrate neural plate in which neighbouring cells can adopt neural or epidermal fates. Curr Biol 10: 869-872.

Cambray, N., V. Wilson (2002) Axial progenitors with extensive potency are localised to the mouse chordoneural hinge. Development 129: 4855-4866. Available at: http://www. ncbi.nlm.nih.gov/pubmed/12361976.

Cambray, N., V. Wilson (2007) Two distinct sources for a population of maturing axial progenitors. Development 134: 2829-2840.

Catala, M., M.A. Teillet, E.M. de Robertis, N.M. Le Douarin (1996) A spinal cord fate map in the avian embryo: while regressing, Hensen's node lays down the notochord and floor plate thus joining the spinal cord lateral walls. Development 122: 2599-2610.

Denans, N., T. Iimura, O. Pourquié (2015) Hox genes control vertebrate body elongation by collinear Wnt repression. eLife 2015: 1-33.

Edri, S., P. Hayward, P. Baillie-Johnson, B. Steventon, A. Martinez Arias (2018) An Epiblast Stem Cell derived multipotent progenitor population for axial extension. bioRxiv DOI: $10.1101 / 242461$.

Fehling, H.J., G. Lacaud, A. Kubo, M. Kennedy, S. Robertson, G. Keller, V. Kouskoff (2003) Tracking mesoderm induction and its specification to the hemangioblast during embryonic stem cell differentiation. Development 130: 4217-4227.

Gouti, M., J. Delile, D. Stamataki, F.J. Wymeersch, Y. Huang, J. Kleinjung, V. Wilson, J. Briscoe (2017) A gene regulatory network balances neural and mesoderm specification during vertebrate trunk development. Dev Cell 41: 243-261.e7.
Gouti, M., A. Tsakiridis, F.J. Wymeersch, Y. Huang, J. Kleinjung, V. Wilson, J. Briscoe (2014) In vitro generation of neuromesodermal progenitors reveals distinct roles for Wnt signalling in the specification of spinal cord and paraxial mesoderm identity. PLoS Biol 12: e1001937.

Hamburger, V., H. Hamilton (1992) A series of normal stages in the development of the chick embryo (reprinted from J Morphol 1951;88: 49). Dev Dyn 195: 231-272.

Henrique, D., E. Abranches, L. Verrier, K.G. Storey (2015) Neuromesodermal progenitors and the making of the spinal cord. Development 142: 2864-2875.

Hooper, M., K. Hardy, A. Handyside, S. Hunter, M. Monk (1987) HPRT-deficient (Lesch-Nyhan) mouse embryos derived from germline colonization by cultured cells. Nature 326: 292-295.

Iimura, T., O. Pourquié (2006) Collinear activation of Hoxb genes during gastrulation is linked to mesoderm cell ingression. Nature 442: 568-571.

Krzywinski, M., N. Altman (2014) Points of significance: visualizing samples with box plots. Nat Methods 11: 119-120.

McGrew, M.J., A. Sherman, S.G. Lillico, F.M. Ellard, P. Radcliffe, H.J. Gilhooley, K. Mitrophanous, N. Cambray, V. Wilson, H. Sang (2008) Localised axial progenitor cell populations in the avian tail bud are not committed to a posterior Hox identity. Development 135: 2289-2299.

New, D.A. (1955) A new technique for the cultivation of the chick embryo in vitro. J Embryol Exp Morphol 3: 320.

Papanayotou, C., A. Benhaddou, A. Camus, A. Perea-Gomez, A. Jouneau, V. Mezger, F. Langa, S. Ott, D. Sabéran-Djoneidi, J. Collignon (2014) A novel nodal enhancer dependent on pluripotency factors and Smad2/3 signaling conditions a regulatory switch during epiblast maturation. PLoS Biol 12: e1001890.

Preibisch, S., S. Saalfeld, P. Tomancak (2009) Globally optimal stitching of tiled 3D microscopic image acquisitions. Bioinformatics 25 : 1463-1465.
Schindelin, J., I. Arganda-Carreras, E. Frise, V. Kaynig, M. Longair, T. Pietzsch, S. Preibisch, C. Rueden, S. Saalfeld, B. Schmid, J.-Y. Tinevez, D.J. White, V. Hartenstein, K. Eliceiri, P. Tomancak, A. Cardona (2012) Fiji: an open-source platform for biological-image analysis. Nat Methods 9: 676-682.

Selleck, M.A., C.D. Stern (1991) Fate mapping and cell lineage analysis of Hensen's node in the chick embryo. Development 112: 615626. Available at: http://www.ncbi.nlm.nih. gov/pubmed/1794328.

Steventon B., A. Martinez Arias (2017) Evo-engineering and the cellular and molecular origins of the vertebrate spinal cord. Dev Biol 432: 3-13.

Tsakiridis, A., Y. Huang, G. Blin, S. Skylaki, F. Wymeersch, R. Osorno, C. Economou, E. Karagianni, S. Zhao, S. Lowell, V. Wilson (2014) Distinct Wnt-driven primitive streaklike populations reflect in vivo lineage precursors. Development 141: 1209-1221.

Turner, D.A., M. Girgin, L. Alonso-Crisostomo, V. Trivedi, P. Baillie-Johnson, C.R. Glodowski, P.C. Hayward, J. Collignon, C. Gustavsen, P. Serup, B. Steventon, M. Lutolf, A.A. Martinez (2017) Anteroposterior polarity and elongation in the absence of extraembryonic tissues and spatially localised signalling in Gastruloids, mammalian embryonic organoids. Development 144: 3894-3906.

Turner, D.A., P.C. Hayward, P. Baillie-Johnson, P. Rue, R. Broome, F. Faunes, A. Martinez Arias (2014) Wnt/ $\beta$-catenin and FGF signalling direct the specification and maintenance of a neuromesodermal axial progenitor in ensembles of mouse embryonic stem cells. Development 141: 4243-4253.

Tzouanacou, E., A. Wegener, F.J. Wymeersch, V. Wilson, J.-F. Nicolas (2009) Redefining the progression of lineage segregations during mammalian embryogenesis by clonal analysis. Dev Cell 17: 365-376.

Voiculescu, O., C. Papanayotou, C.D. Stern (2008) Spatially and temporally controlled electroporation of early chick embryos. Nat Protoc 3: 419-426.

Wymeersch, F.J., Y. Huang, G. Blin, N. Cambray, R. Wilkie, F.C. Wong, V. Wilson (2016) Position-dependent plasticity of distinct progenitor types in the primitive streak. eLife $5: 1-28$. 